\title{
Journal for immunotherapy of cancer reviewer acknowledgement 2015
}

Pedro Romero

\section{Contributing reviewers}

On behalf of the JITC Section Editors and Associate Editors, I wish to acknowledge with sincere appreciation the assistance of the following reviewers who generously contributed their time and efforts in 2015 in the appraisals of manuscripts submitted to the journal. These reviewers not only have been invaluable in assessing the merit of submitted studies but also, by their careful analysis and critique and their general and specific constructive recommendations, have often greatly enhanced the value of these manuscripts. The quality of JITC can be attributed in large measure to their dedication and we are sincerely grateful.

\author{
Carlos Alfaro \\ Spain
}

Ramon Arens

Netherlands

Shailender Bhatia
USA

Christian Blank

Netherlands

Marie Bleakley

USA

Renier Brentjens

USA

Martin Cannon

USA

Paola Cappello

Italy

John Castle

Germany

Ugo Cavallaro

Italy

Bartosz Chmielowski

USA
Joe Clark

USA

\begin{abstract}
Alice Conlin
USA
\end{abstract}

\section{Bruno Correia \\ USA}

Todd Crocenzi

USA

\section{Brendan Curti}

USA

Toos Daemen

Netherlands

\section{Patrick Dillon \\ USA}

Daniela Dinulescu

USA

Reinhard Dummer

Switzerland

Janice Dutcher

USA

Lea Eisenbach

Israel

\author{
Zhichao Fan \\ USA
}

Mark Faries

USA
Morganna Freeman-Keller
USA

Claus Garbe

Germany

Ulrike Gnad-Vogt

Germany

John Godwin
USA

\section{Gullu Gorgun \\ USA}

John Haanen

Netherlands

M Heemskerk

Netherlands

Peter Hersey

Australia

Rafat Husain

USA

Correspondence: immunotherapyofcancer@biomedcentral.com

Ludwig Center for Cancer Research, University of Lausanne, Lausanne,

Switzerland 
Theodore Johnson

USA

Harriet Kluger

USA

Viktor Koelzer

Switzerland

Holbrook Kohrt

USA

Lawrence Lamb

USA

Heinz Läubli

Switzerland

Peter Lee

USA

Qiao Li

USA

Devin Lowe

USA

Jason Luke

USA

Jose Lutzky

USA
Giuseppe Masucci

Sweden

Jeffrey Miller

USA

Christian Munz

Switzerland

Thorunn Olafsdottir

Sweden

Eileen O'Reilly

USA

Patrick Ott

USA

David Page

USA

Julian Pardo

Spain

Manish Patankar

USA

Sapna Patel

USA

Jose Luis Perez Gracia

Spain
Shari Pilon-Thomas

USA

John Powderly

USA

Daniel Powell

USA

Ryan Sullivan

USA

Paul Tumeh

USA

Dominique Vanhecke Switzerland

John Vetto

USA

Michael Von Bergwelt

Germany

Derek Wainwright

USA

Hassane Zarour

USA

Jacques Zimmer

Luxembourg 